\title{
Comparison of normalized gain and Cohen's d for Force Concept Inventory results in an introductory mechanics course
}

\author{
David Donnelly, ${ }^{1}$ Jean-Michel Mailloux-Huberdeau, ${ }^{1}$ Jayson M. Nissen, ${ }^{2}$ and Eleanor W. Close ${ }^{1}$ \\ ${ }^{1}$ Physics Department, Texas State University, 601 University Dr., San Marcos, TX, 78666 \\ ${ }^{2}$ Science Education Department, CSU Chico, 101 Holt Hall, Chico, CA, 95929
}

\begin{abstract}
At Texas State University, we have been using the Force Concept Inventory (FCI) to assess our introductory mechanics course since the Spring 2011 semester. This provides us with a large data set $(\mathrm{N}=1,626)$ on which to perform detailed statistical analysis of student learning. Recent research has found conflicting results in the relationships between normalized gain $\langle g\rangle$, Cohen's $d$, and pretest mean, which might lead to different interpretations of student learning. Specifically, in one study $\langle g\rangle$ was found to positively correlate with both pretest mean and pretest standard deviation, whereas Cohen's $d$ did not; in another study, ANOVA showed no connection between $\langle g\rangle$ and pretest mean. We will present a comparison of $\langle g\rangle$ and Cohen's $d$ for our data set, and will specifically use these measures to look at performance gaps related to gender and race/ethnicity.
\end{abstract}

Keywords: Concept Inventory, Effect Size, Normalized Gain

\section{INTRODUCTION}

Texas State University is a minority serving institution with an enrollment of approximately 39,000 students in the fall, 2016 semester. The calculus-based mechanics course in the Physics Department serves Physics, Engineering, and Chemistry majors. Starting in the Spring 2011 semester, the Force Concept Inventory (FCI) [1] has been used to assess student conceptual learning in the course. The goal of administering this concept inventory was to assess the degree to which students were gaining conceptual understanding in the course, and to inform how structural changes in the course were impacting student outcomes. For example, we used the FCI to assess the impact of a Learning Assistant Program that was piloted in Spring 2012 and expanded to all course sections in Fall 2012. Apart from the implementation of the Learning Assistant Program and changes in instructional staff, no other changes to the course have been made.

In total, we have matched data for 1,626 students spread over 12 semesters. Traditionally, $\langle g\rangle$ [2] has been used to measure changes in student conceptual understanding between pre- and posttests, and that is the metric we have been using. However, some researchers $[3,4]$ have begun advocating for the use of some measure of effect size other than $\langle g\rangle$. Use of effect size measures, such as Cohen's $d$, allows for comparison of results using different instruments, or, relevant for this study, between different populations. This suggests that Cohen's $d$ might be a better measure to use when comparing the learning of different populations within the same course. In this paper, we will analyze our data using both $\langle g\rangle$ and Cohen's $d$, and discuss how the two measures might lead to different conclusions about equity with regard to gender and ethnicity.

\section{II.RESEARCH QUESTIONS}

The purpose of this work is to analyze our data to determine if the introductory mechanics course provides equal learning opportunities for all students, and what the best metric is to assess this. The primary research question for this paper is whether the uses of an effect size measure such as Cohen's d lead to different conclusions than $\langle g\rangle$ when analyzing learning gains by different groups in the same class. To answer this question we will look at FCI scores for female students vs. male students using both $\langle g\rangle$ and $\mathrm{d}$. We will also compare the scores of students from overrepresented ethnic/racial groups to those of students from underrepresented ethnic/racial groups.

\section{III.DATA ACQUISITION AND ANALYSIS}

The FCI was administered in a pre/post format, with the pretest being given very close to the start of the semester, and the posttest being given some time after completion of instruction on Newton's Laws. For most semesters, the assessment was completed using paper and pencil either in class or in lab. Starting in Spring, 2016, online administration has been an option using the LASSO platform hosted by the Learning Assistant Alliance. Using this tool, some instructors have administered the instrument online, while others have continued administering it in class. Demographic data was gathered after the test was administered using student identification numbers

Once data were obtained, they were filtered so that only students who had both a pretest and posttest score were used 
in the analysis. $\langle g\rangle$ was calculated using the standard formula [2].

$$
\langle g\rangle=\frac{\bar{x}_{\text {post }}-\bar{x}_{\text {pre }}}{100 \%-\bar{x}_{\text {pre }}}
$$

Here $\bar{x}$ represents the mean score on either the pre- or posttest. Data are analyzed by semester, semesters are labeled by calendar year, so, for example, 2012.1 would refer to the first semester in calendar year 2012. When we were analyzing different groups, the means for those groups for each semester were used. There are other methods one can use to calculate gain, but these methods show strong correlation with the method described above [4]. Error bars on graphs of $\langle g\rangle$ are $95 \%$ Confidence Intervals, and are calculated using the Standard Error calculation described by Smith et al. [5].

To calculate Cohen's d, we used the effsize package in R, which employs the formula

$$
d=\frac{\bar{x}_{\text {post }}-\bar{x}_{\text {pre }}}{s}
$$

where $\mathrm{s}$ is the pooled standard deviation of the pre- and posttests. Error bars on graphs of d are also $95 \%$ Confidence Intervals.

When comparing female to male students, students from each semester were separated by gender, and $\langle g\rangle$ and Cohen's $d$ were calculated for each group for each semester. For comparing students in underrepresented racial or ethnic groups to those in overrepresented groups, we broke students up into two groups: overrepresented (White and Asian, $\sim 46 \%$ of students in Fall 2016) and underrepresented (all other groups, $\sim 54 \%$ of students in Fall 2016). Of the students from underrepresented groups, Hispanic ( $35 \%$ in Fall 2016) and Black ( $11 \%$ in Fall 2016) students comprised most of the group, with small numbers of students from other races/ethnicities.

Whenever equity is discussed, one must be clear what equity model is being used [3]. In our course, we use an equity of fairness model, with the goal that all students achieve the same learning gains, regardless of pretest score.

\section{IV.RESULTS}

For brevity we have not included tables of the data. In the interest of full disclosure and reproducibility, the data tables are available from the first author. $\langle g\rangle$ for female and male students is shown in Fig. 1, and Cohen's $d$ for the two groups is shown in Fig. 2. When $\langle g\rangle$ is used as a metric, male students show larger conceptual gains than female students in all but one of the 12 semesters studied. However, when
Cohen's $d$ is used, the reverse is true: female students show larger gains than male students in all but three semesters. For $\langle g\rangle$, mean values for male students are outside of the $95 \% \mathrm{CI}$ for female students in most semesters. This indicates that there is a reliable difference in $\langle g\rangle$ between male and female students. For $d$, however, values for male students all fall within the $95 \%$ CI of the female student values, indicating the difference is not reliable. Consequently, $\langle g\rangle$ indicates that our courses are consistently and reliably inequitable and $\langle g\rangle$ also seems to indicate that the more effective the courses are the more inequitable they become. In contrast, Cohen's $d$ indicates that the effects on female students were similar in size to those on male students and were not reliably different.

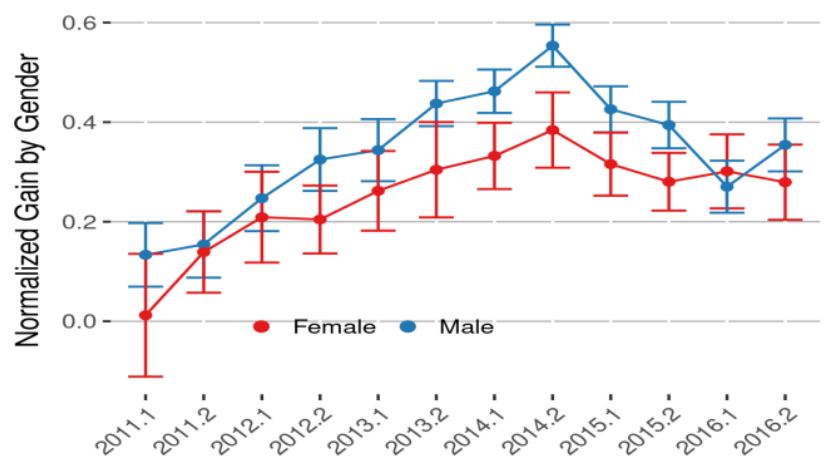

FIG 1. Normalized gain $\langle g\rangle$ by semester for male and female students.

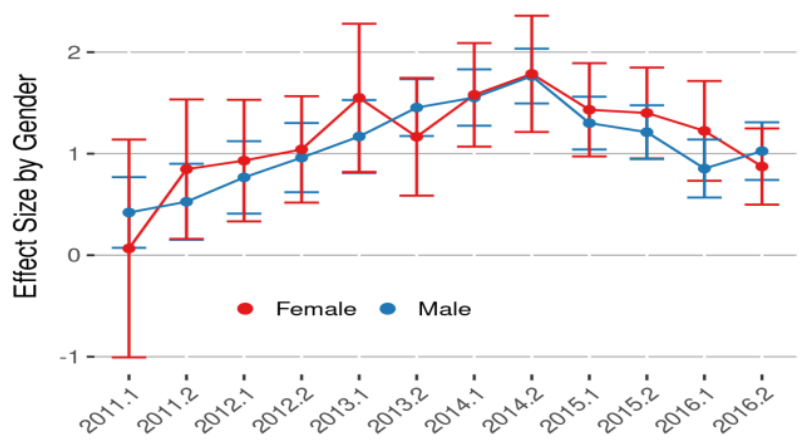

FIG 2. Cohen's $d$ by semester for male and female students.

$\langle g\rangle$ for students from underrepresented vs. overrepresented race/ethnicity groups is shown in Fig. 3, and Cohen's $d$ for the two groups is shown in Fig. 4. When $\langle g\rangle$ is used as a metric, students from the overrepresented group outperform students from the underrepresented group for all semesters studied. However, when Cohen's $d$ is used as a metric, students from overrepresented groups outperform students from underrepresented groups only prior to Fall 2014. Beginning in Fall 2014, both groups have approximately equal performance. As with the gender analysis, for $\langle g\rangle$, mean values for students from 
overrepresented groups are outside of the $95 \%$ CI of students from underrepresented groups for 10 of the 12 semesters. This indicates that there is a reliable difference in $\langle g\rangle$ between the two groups. For $d$, however, values for students from overrepresented groups fall within the $95 \% \mathrm{CI}$ of the values of students from underrepresented groups, with one exception, indicating the difference is not as reliable. Thus, $\langle g\rangle$ indicates that there is a reliable inequity in the courses, though this inequity for race/ethnicity does not seem to increase as the overall effectiveness of the course increases. Cohen's $d$ also indicates that there is a small inequity for semesters prior to Fall 2014, but the overlap in Confidence Intervals indicates that this is not a reliable inequity.

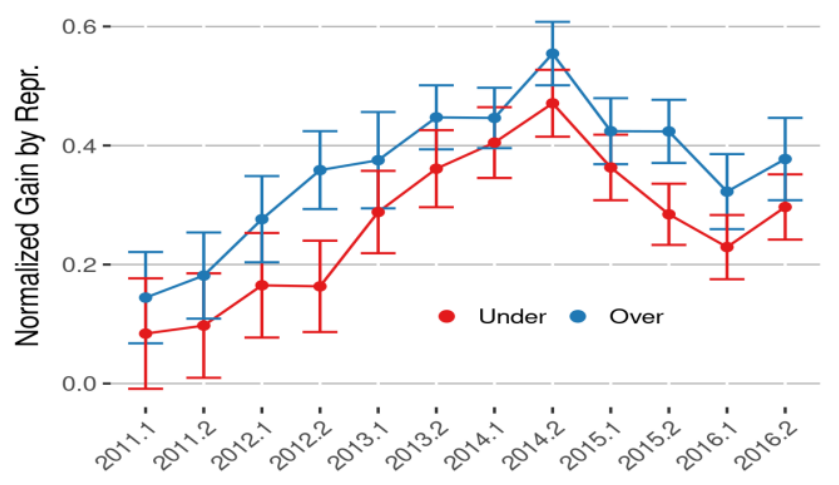

FIG 3. Normalized gain by race/ethnicity representation.

\section{V.DISCUSSION}

In an effort to understand the differences obtained when using $\langle g\rangle$ vs. Cohen's $d$, we subsequently looked at mean scores on both pre- and posttest for each semester that data was collected. These data are shown in Fig. 5 and Fig. 6. In every semester for which we have data, both the pretest mean and posttest mean for female and underrepresented students were lower than those for male students and overrepresented students, respectively. Similar results have been reported by other researchers [6].

The difference between male and female students' pretests scores varied but was 3.5 questions averaged over the 12 semesters. Differences in pretest scores for over and underrepresented populations were on average only 2 questions. This smaller pretest difference for race/ethnicity may, in part, explain why the results of $\langle g\rangle$ and Cohen's $d$ agreed more for race/ethnicity than they did for gender.

When comparing $\langle g\rangle$ to effect size, there are several factors that need to be considered. $\langle g\rangle$ has been defined in such a way that in two populations with the same raw gain, the population with the higher pretest mean will have a higher $\langle g\rangle$. In other words, $\langle g\rangle$ is trying to account for a ceiling effect that would make it more difficult for highpretest populations to have high gains. For Cohen's $d$, both raw gain and pooled standard deviation contribute to the effect size.

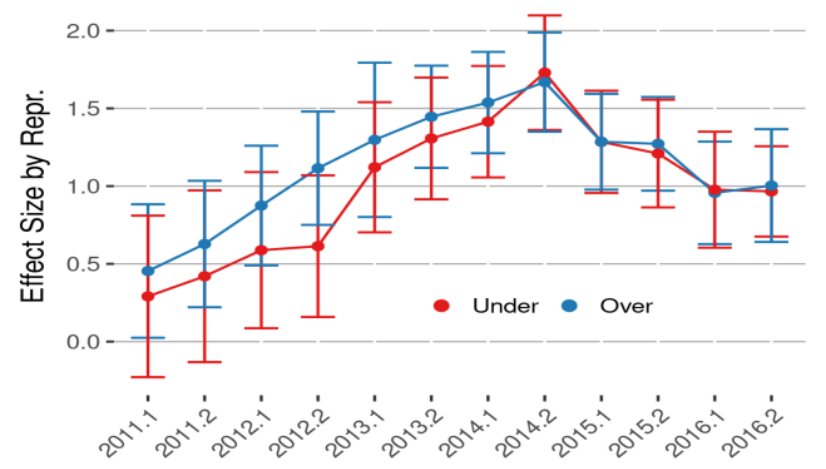

FIG 4. Cohen's $d$ by race/ethnicity representation.

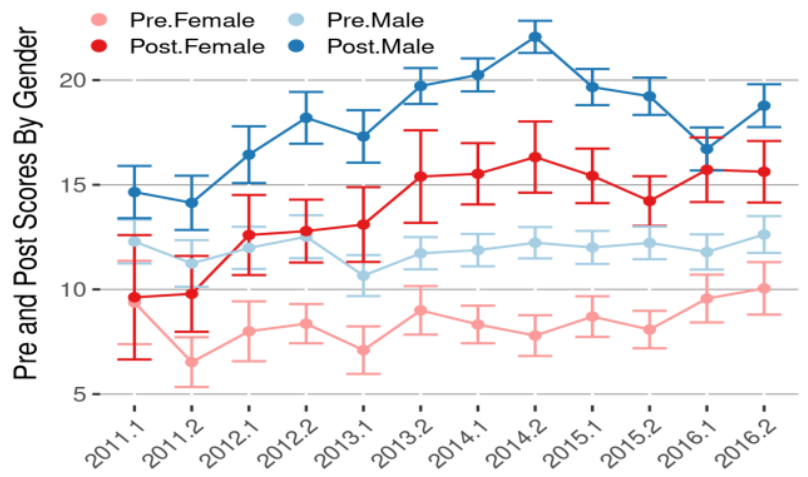

FIG 5. Pretest and posttest mean scores by gender.

The use of standard deviation generally means that the impact of outliers on Cohen's $d$ will be mitigated because while they increase the means they also increase the pooled standard deviation.

However, the specific case of a single outlier high score on the pretest for a small sample of students can have an amplified dampening effect on Cohen's $d$ for that population of students because the outlier increases both the pretest mean and the pooled standard deviation. Both of these effects act to decrease the size of Cohen's $d$. For example, in Spring 2011 there were matched data from only 8 female students. If one of these students scored relatively high on the pretest it would reduce the effect size, and also increase the size of the confidence intervals. We believe that these "outlier" effects might explain some of the variation we see in the effect size for female students. This also explains why the error bars for Spring 2011 are so large.

Depending on which metric is used, analysis of these data suggest contradictory findings regarding the equity of this course, with regard to both gender and race/ethnicity. Using $\langle g\rangle$ would lead to the conclusion that we had not achieved the desired level of equity of fairness in the class. When female students are compared to male students, normalized gain is lower in 11 of the 12 semesters studied. 


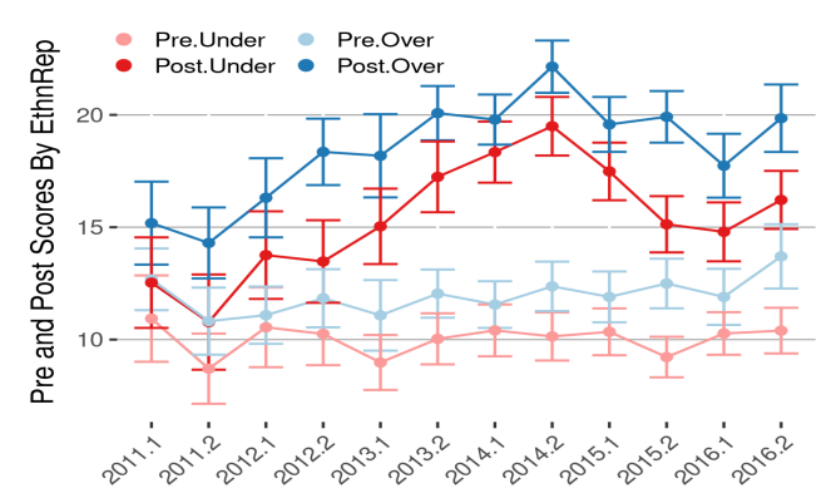

FIG 6. Pretest and posttest mean scores by ethnic/racial representation.

When students from underrepresented groups are compared to those from overrepresented groups, $\langle g\rangle$ is lower in all 12 semesters, for the majority of these semesters $\langle g\rangle$ indicated that these differences were statistically reliable. On the other hand, Cohen's $d$ indicates that we have achieved equity of fairness when comparing male and female students. Effect sizes for females were slightly higher in 9 of the 12 semesters and there is large overlap in the confidence intervals in each semester, indicating at the very least that we cannot reliably say that the course was inequitable. In the case of students from over- and underrepresented groups, we see similar results, $\langle g\rangle$ indicated a consistent inequity that was reliable, in contrast with Cohen's $d$ which indicated that there was an inequity prior to 2014 that was not always reliable though it was often quite large but that in recent semesters this inequity had largely disappeared.

There are limitations to using Cohen's $d$. It is well known that Cohen's $d$ over-estimates the effect size, particularly for small samples of students. This over-estimation may have had a larger effect in our study on the analysis by gender than by race/ethnicity because female students made up a much smaller fraction of the data than underrepresented students. In this study, we used Cohen's $d$ because it is widely used in other studies in both physics and education. In our future work we will also use Hedge's $g$, which compensates for the overestimation that Cohen's $d$ produces. It is also important to note that we do not know the limitations of $\langle g\rangle$. While many studies have investigated the properties of Cohen's $d$ we are not aware of any similar body of work investigating the validity of $\langle g\rangle$.

[1] D. Hestenes, M. Wells, and G. Swackhamer, Phys. Teach., 30 (3), 141 (1992)

[2] R. Hake, American Journal of Physics, 66(1):64 (1998)

[3] I. Rodriquez, E. Brewe, V. Sawtelle, and L. Kramer, Phys. Rev. ST Phys. Educ. Res., 8, 020103 (2012)
The results of this snalysis are of value to the broader Physics Education Research Community as they indicate that care must be taken when comparing Conceptual Inventory gains between different groups in the same course. When comparing different populations for whom pretest mean, posttest mean, and standard deviation might be different, effect size provides a measure that avoids bias toward higher pretest means. It is also important to note that differences in Concept Inventory scores are not the ultimate measure of equity, however they are often an entry point for instructors to begin measuring equity in their own courses, and thus they play an important role in how physics educators think about and act toward issues of equity in physics.

To answer the question about which metric should be used to evaluate a particular data set, we feel that effect size metrics are always preferable to normalized gain. However, if a researcher is only looking at changes in conceptual gain for an entire class, and pretest mean for that class is stable over time, then normalized gain will provide essentially the same information as an effect size measure such as Cohen's $d$. If one is comparing different groups within the same class, effect size measures are preferred, although one must be careful to account for effects due to small data sets.

Future work will utilize data from students for whom either a pre or posttest score is missing. We will use Multiple Imputations to impute the missing data instead of listwise deletion of unmatched data. The use of only matched data assumes that all missing data is missing at random and can thus be ignored. To our knowledge the PER community has never tested this assumption even though it seems reasonable to assume that students who provide complete data sets are more likely to be high-performing students. Thus, the lower the participation rates the more elite the measured sample is likely to be. This is particularly relevant to the course investigated in this study as the integration of LAs into the course coincided with a dramatic decrease in the DFW rates in the course and a focus on improved data collection.

\section{ACKNOWLEDGEMENTS}

This work and the Texas State University LA Program are supported in part by NSF Grant Nos. 1240036, 1557405, 0808790, and 1431578, and by the Halliburton Foundation. 